\title{
Design and Analysis of a Wireless Link Control Scheme for Integrated
}

\section{Service Communications}

\author{
Hung-Yi Chen* and Jin-Fu Chang** \\ * Department of Electrical Engineering, National Taiwan University, Taipei, Taiwan 10617, R.O.C. \\ ** National Science Council, Taipei, Taiwan 10636, R.O.C. \\ E-mail: chy@fcom.ee.ntu.edu.tw, jfchang@nsc.gov.tw
}

\begin{abstract}
In this paper, a wireless link control scheme counteracting correlated channel errors for multimedia type communications is proposed. In the proposed scheme, both the medium access (MAC) protocol and data link control (DLC) protocol are addressed. Performance is analyzed for the well-known Gilbert correlative channel. Performance measures obtained include the cell loss probabilities for the CBR and VBR cells, mean delay and delay jitter for the VBR cells, and the throughput for the ABR cells. The impact of system parameters on the system performance is also investigated. Numerical examples are given to demonstrate that our proposed scheme is able to meet the quality-ofservice guarantees required by the multimedia type communications.
\end{abstract}

\section{Introduction}

Asynchronous transfer mode (ATM) has been adopted by the International Telecommunication Union (ITU) for the broadband integrated service digital network (BISDN) [1]. Moreover, in order to provide seamless service, the extension of ATM technology to the wireless medium has been actively pursued and is widely known as the wireless ATM (WATM). For the WATM, medium access control (MAC) and data link control (DLC) are established to facilitate channel sharing and error control on the radio links. When properly designed, they ought to be able to provide QoS guarantee as the wired ATM. Several MAC protocols of TDMA/FDD type for the
WATM have been proposed in the literature [2]-[4]. These protocols address resource reservation and scheduling but neglect error control. In [5]-[7], the data link layer is included to provide error protection. In this paper, we propose a wireless link control scheme to meet different QoS requirements and to also counteract the correlated errors occurring in the wireless environment. Although the frame structure is similar to [4], we have modified it to become more flexible and efficient in supporting different QoS needs.

\section{Protocol}

In the protocol, two aspects are addressed: MAC and DLC. The main function of a MAC protocol is to support channel sharing among various traffic types. A DLC protocol is designed to mitigate the effect of channel errors.

\section{A. The MAC Protocol}

Like many other studies, we only concentrate on the uplink. The uplink frame structure of the proposed access scheme is shown in Fig. 1. The channel time is divided into slots and grouped into frames; the slot duration is equivalent to the cell transmission time. Each uplink frame is further divided into three subframes: request, reservation, and retransmission. The boundaries between these subframes are assumed fixed. The request subframe consists of a number of minislots. Since the 
CBR and VBR traffic have different desires for QoS, it is convenient to divide the request subframe into two parts: the CBR request area and the VBR request area. A piggyback field is appended at the end of each VBR cell to inform the base station to assign additional slots. In the reservation subframe, the slots that are not reserved will be converted by the base station into minislots to become the request area for the ABR users. The base station again broadcasts a message at the end of the reservation subframe to inform the VBR users which cells need to be retransmitted and how many extra new cells can be transmitted in the retransmission subframe. Retransmitted cells are given priorities over extra new cells to access the retransmission subframe. After having assigned slots to retransmitted cells and extra cells, if there are still slots available in the retransmission subframe, extra cells may be transmitted for a second time for protection. If there are still slots remaining in the retransmission subframe, they are assigned to the ABR users. Cells without chances to get transmission in the current frame are dropped.

An ABR user after having made a successful reservation, traffic slots are assigned according to its demand and the availability of slots. An ABR user releases the ownership after the frame ends. Slots assigned to the ABR users are arranged in a dynamic manner. The minimum cell rate (MCR) is always guaranteed for the ABR users having successful reservation.

\section{B. DLC Protocol}

A combination of FEC with finite interleaving and ARQ is used for error control. Since the CBR traffic requires a rigid delay constraint, no retransmission is considered. Regarding the VBR traffic, the combination of SR and partial stutter is employed. The readers may consult [8] for the meaning of stutter. The reserved VBR cells having failed transmission are required to be in the SR mode. After having assigned slots to retransmitted cells and extra cells, an extra VBR cell has the $0-7803-5718-3 / 00 / \$ 10.00 @ 2000$ IEEE. opportunity to be sent again if there are still available slots. Since not all extra VBR cells always have such opportunities, these extra cells are said to be in the partial stutter mode. Regarding the ABR users, cells having failed transmission are placed in the SR mode till they succeed.

\section{Channel Model and Error Control Scheme}

The Gilbert channel model [9] employed in this paper is shown in Fig. 2. In the "Good" state, errors occur with probability $P_{G}$ while in the "Bad" state, errors occur with probability $P_{B}$. It is reasonable to assume that $P_{G} \ll P_{B}$. If interleaving with degree $I$ is considered, the transition matrix has been derived in [10] as follows:

$$
\mathbf{P}=\left[\begin{array}{cc}
p\left(1-\rho^{\prime}\right) & 1-p\left(1-\rho^{i}\right) \\
1-(1-p)\left(1-\rho^{\prime}\right) & (1-p)\left(1-\rho^{l}\right)
\end{array}\right]
$$

where $\rho=1-\alpha-\beta$ and $p=\alpha / \alpha+\beta$.

A type-I hybrid ARQ using Reed-Solomon code for error control over wireless channel has been employed in the literatures [11]. From [10] and [12], the bit error probability after decoding can be obtained as follows:

$$
P_{e b}=\frac{2^{m-1}}{n-1} P_{e s}
$$

\section{Traffic Models}

We use the ON-OFF model to characterize the CBR and VBR traffic sources [13], [14]. The periods of $O N$ and OFF are assumed to be exponentially distributed. When a CBR user becomes active, a fixed number of cells are generated per frame. For an active VBR user, the number of cells generated in a frame becomes a variable described by the probability distribution function $Q_{v}=\left\{q_{v}^{1}, q_{v}^{2}, \cdots, q_{r}^{p_{1}}\right\}$, where $q_{V}^{i}$ is the probability of generating i cells in a frame, and $p_{v}$ is the maximum number of cells that can be generated by a VBR source in a frame. We assume that the ABR users always have cells to compete for the residual 
resource not used by the CBR and VBR community.

\section{Performance Analysis}

In our proposed protocol, since the CBR and VBR users having made a successful request reserve a fixed amount of traffic slots in the succeeding frames, we employ the approach used in [4] to analyze the performance of the CBR and VBR traffic in our system. For the CBR and VBR traffic, the interested performance measures are the cell loss probability, cell delay, jitter, and cell residual error probability. For the ABR traffic, only throughput is considered here. Although details are not given here due to page limitation, the authors will provide them upon request.

The cell loss probability is defined to be the average fraction of cells dropped during the $\mathrm{ON}$ period due to delayed transmission. The delay suffered by a cell is measured from the beginning of the frame it is scheduled till the time its transmission finishes. Because of the micro- or pico-cell system considered in this paper, the propagation delay is considerably small and can be neglected. Since a CBR cell has a fixed slot in the frame for transmission, its delay is not only bounded but also fixed. For a VBR cell, however, its delay remains bounded but exhibits more variance. Following [15], we define jitter as the variance of delay.

The residual error probability of a cell is defined to be the probability that a cell accepted by the receiver contains errors. For the CBR traffic, since retransmission is not considered, the residual error probability of a CBR cell is equal to the actual cell error probability.

Since the ABR traffic is loss-sensitive, cells are stored in the buffer till they are transmitted successfully. We assume that each $A B R$ user has a buffer large enough so that overflow does not occur. The throughput of the ABR traffic is defined to be the average number of cells that an $A B R$ user can send through in a frame.

\section{Numerical Results and Discussions}

We use the shortened Reed-Solomon $(69,53,8)$ with 0-7803-5718-3/00/\$10.00 O2000 IEEE. interleaving degree of 8 to encode 8-bit symbols for FEC. The channel parameter $\rho$ in (1) is set to 0.9 . The probability mass function of the cell generation rate per frame of a VBR user is given in Table1. From Tables 2 and 3, we observe that if a cell loss probability for the CBR traffic is required to be in the order of $10^{-6}$, the number of request slots allocated to the CBR users appears to be approximately one half of the number of CBR users. For the VBR traffic, the number of request slots assigned to the VBR users is roughly equal to the number of VBR users in the system if a cell loss probability of $10^{-7}$ is desired.

The relation between the traffic source ON-OFF periods and the cell loss probability is examined in Table 4, where we show that the longer source ON period, the lower cell loss probability appears. There exists a trade-off between the number of retransmissions and the residual error probability. For the CBR and VBR traffic, the residual cell error probability appears to be larger than the ABR traffic due to the delay constraint. The residual cell error probability of the VBR cells is plotted in Fig. 3. It is obvious that the residual cell error probability of the VBR cells is lower than the CBR cells because of the ARQ scheme employed. The residual cell error probability is larger if only the minimum cell rate is allocated. Using (2), the BER after decoding is plotted in Fig. 4. In our proposed protocol, the BER after decoding is in the order of $10^{-4}$. In [16], it is suggested that a BER of $10^{-4}$ measured at the top of the PHY layer be required.

The delay of the VBR cells under different values of $n_{v}$ is plotted in Fig. 5. We observe that delay of the VBR cells decreases as the value of $n_{\psi}$ increases. In addition, it is shown that delay behavior appears immune to the channel condition. The reason is that the FEC used in our proposed protocols is effective enough to remove the errors occurred so that the cell delay is not much affected by the retransmission. Similar phenomenon can be observed in Fig. 6 regarding jitter of the VBR cells. From Tables 5-7, we observe that the number of available traffic slots for the ABR traffic decreases as the value of $n_{y}$ increases. In addition, it is evident to see that the throughput of the ABR traffic stays rather unchanged

VTC2000 
regardless of the value of $n_{V}$.

\section{Conclusions}

In this paper, we have proposed a wireless link control scheme to support integrated services over the wireless links. We have analyzed the performance of the proposed scheme in the paper. Numerical results have been provided to demonstrate that the proposed scheme can meet the QoS requirement mandated by the multimedia communication requirement.

\section{REFERENCE}

[1] W. Stallings, High-Speed Networks: TCP/IP and ATM Design Principles, Prentice-Hall Inc., 1998.

[2] J. G. Kim and I. Widjaja, "PRMA/DA: A New Media Access Control Protocol for Wireless ATM," Proc. ICC'96, pp.11-19.

[3] M. J. Karol, Z. Liu, and K. Y. Eng, "DistributedQueuing Request Update Multiple Access (DQRUMA) for Wireless Packet (ATM) Networks." Proc. IEEE INFOCOM'95, pp. 1224-1231.

[4] X. Qiu, O. K. Li, and J.-H. Ju, "A multiple access scheme for multimedia traffic in wireless ATM," ACM Mobile Networks and Applications, vol. 1, pp. 259-272, 1996.

[5] H. Xie et al., "Data Link Control Protocols for Wireless ATM Access Channels," Proc: ICUPC'95, pp.1-5, Nov. 1995.

[6] F. Bauchot et al., "MASCARA, a MAC Protocol for Wireless ATM," Proc. ACTS Mobile Summit'96, Granada, Spain, pp. 17-22, Nov. 1996.

[7] F. D. Priscoli, "Medium Access Control for the MEDIAN System," Proc. ACTS Mobile Summit'96, Granada, Spain, pp. 1-8, Nov. 1996.

[8] M. J. Miller et al., "The analysis of some selective repeat ARQ schemes with finite buffer," IEEE Trans. Commun., vol. 29, pp.1307-1315, Sept. 1981.

[9] E. N. Gilbert, "Capacity of a Burst Noise Channel,"
Bell System Technical Journal, vol. 39, pp. 1253-1265, Sept. 1960.

[10] J. R. Yee et al., "Evaluation of the Performance of ErrorCorrecting Codes on a Gilbert Channel," IEEE Trans. Commun., vol. 43, no. 8, Aug. 1995.

[11] S. B. Wicker, "Reed-Solomon Error Control Coding for Rayleigh Fading Channels with Feedback," IEEE Trans. Veh. Tech., vol. 41, pp. 124-133, May 1992.

[12] J. G. Proakis, Digital Communications, $3^{\text {rd }}$ ed., McGRAW-Hill Inc., 1995.

[13] P. Narasimhan and R. D. Yates, "A new protocol for the integration of voice and data over PRMA," IEEE J. Select. Area Commun., vol. 14, no. 4, pp. 623-631, May 1996.

[14] D. P. Heyman et al., "Statistical analysis and simulation study of video teleconference traffic in ATM networks," IEEE Trans. Circuits and Syst. for Video Tech. 2, pp. 49-58, 1992.

[15] H. Saito, Teletraffic Technologies in ATM Networks, Artech House, Boston, London, 1994.

[16] The ATM Forum-Baseline Text for Wireless ATM specifications, 1997.

Table 1. Probability distribution of the cell generation rate

per frame of a VBR source
\begin{tabular}{|c|c|c|c|}
\hline $\mathrm{i}$ (cells/frame/user) & 1 & 2 & 3 \\
\hline$q_{V}^{i}$ & 0.2 & 0.5 & 0.3 \\
\hline
\end{tabular}

Table 2. Number of request slots assigned to the CBR users v.s. the number of CBR users

\begin{tabular}{|c|c|c|}
\hline No. of CBR user & No. of request slots a & Cell Loss Probability \\
\hline 4 & 2 & $1.2769^{*} 10^{-6}$ \\
\hline 5 & 3 & $8.5102^{*} 10^{-7}$ \\
\hline 7 & 4 & $8.5112^{*} 10^{-7}$ \\
\hline
\end{tabular}

Table 3. Number of request slots assigned to the VBR users

v.s. the number of VBR users
\begin{tabular}{|c|c|c|}
\hline No. of VBR users & No. of request slots assign & Cell Loss Probability \\
\hline 4 & 4 & $1.0635^{*} 10^{-7}$ \\
\hline 5 & 5 & $1.0636^{*} 10^{-7}$ \\
\hline 7 & 7 & $1.0636^{*} 10^{-7}$ \\
\hline
\end{tabular}


Table 4. Relation between the source period and the cell loss \begin{tabular}{l|l|l|l}
$(1.0,1.35) \rightarrow(\mathrm{a}, \mathrm{b})$ & $(\mathrm{a}, \mathrm{b})=(1.0,5.4)$ & $(\mathrm{a}, \mathrm{b})=(3.0,2.7)$ & $(\mathrm{a}, \mathrm{b})=(4.0,5.4)$ \\
\hline
\end{tabular} Ratio of the ON period $(a / l)$

Ratio of the cell

loss probability

0.0625

Table 5. Throughput of the ABR traffic as $n_{v}=1$

\begin{tabular}{|c|c|c|c|}
\hline$P_{\text {cell }}^{c}$ & $10^{-3}$ & $5^{*} 10^{-3}$ & $10^{-2}$ \\
\hline No. of available traffic slots & 8.2615 & 8.2601 & 8.2567 \\
\hline No. of reserved ABR users & 6.2766 & 6.2759 & 6.2742 \\
\hline Throughput & 1.3162 & 1.3162 & 1.3160 \\
\hline
\end{tabular}

Table 6. Throughput of the ABR traffic as $n_{v}=2$

\begin{tabular}{|c|c|c|c|}
\hline$P_{c e l l}^{c}$ & $10^{-3}$ & $5^{*} 10^{-3}$ & $10^{-2}$ \\
\hline No. of available traffic slots & 7.4009 & 7.3996 & 7.3963 \\
\hline No. of reserved ABR users & 4.9941 & 4.9938 & 4.9931 \\
\hline Throughput & 1.4819 & 1.4818 & 1.4813 \\
\hline
\end{tabular}

Table 7. Throughput of the ABR traffic as $n_{v}=3$

\begin{tabular}{|c|c|c|c|}
\hline$P_{\text {cell }}^{e}$ & $10^{-3}$ & $5^{*} 10^{-3}$ & $10^{-2}$ \\
\hline No. of available traffic slots & 5.2229 & 5.2226 & 5.2220 \\
\hline No. of reserved ABR users & 3.6934 & 3.6934 & 3.6933 \\
\hline Throughput & 1.4141 & 1.4140 & 1.4139 \\
\hline
\end{tabular}

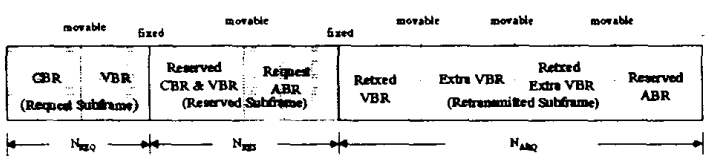

Fig. 1 The uplink frame structure of the protocol

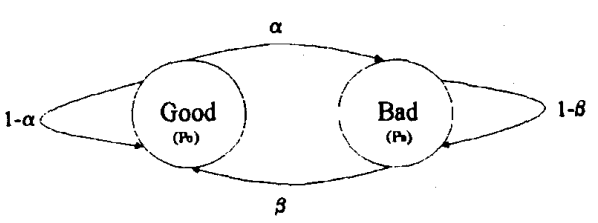

Fig. 2 The Gilbert channel model

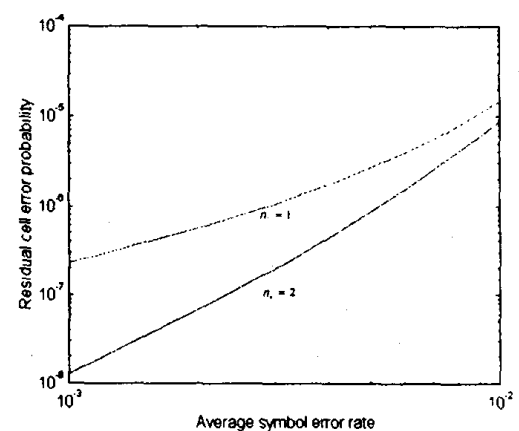

Fig. 3 Residual cell error probability of the VBR cells

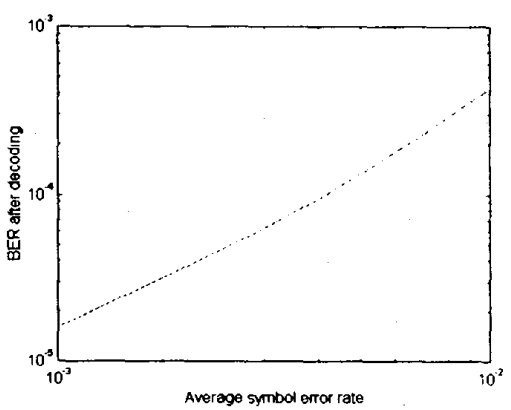

Fig. 4 BER after decoding

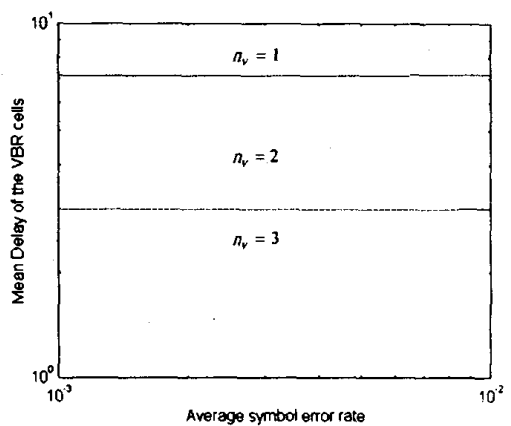

Fig. 5 Mean delay of the VBR cells

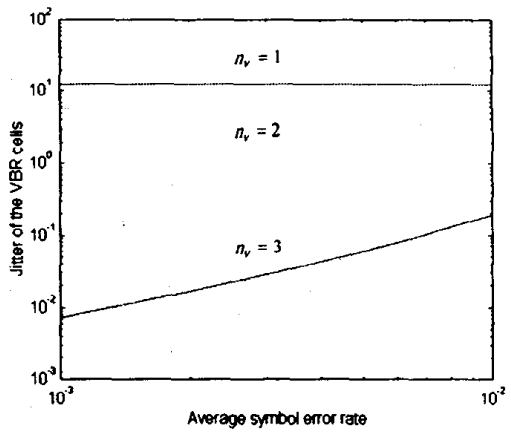

Fig. 6 The jitter of the VBR cells 